A N N A L E S Annales de Bretagne et des Pays de l'Ouest

\title{
Le parlement de Bretagne et la réglementation du port d'armes (1554-1789)
}

The Breton Parlement and Regulating the Carrying of Weapons (1554-1789)

Julien Le Lec

\section{CpenEdition}

Édition électronique

URL : http://journals.openedition.org/abpo/3121

DOI : 10.4000/abpo.3121

ISSN : 2108-6443

Éditeur

Presses universitaires de Rennes

\section{Édition imprimée}

Date de publication : 31 octobre 2015

Pagination : 130-149

ISSN : 0399-0826

\section{Référence électronique}

Julien Le Lec, "Le parlement de Bretagne et la réglementation du port d'armes (1554-1789) », Annales de Bretagne et des Pays de l'Ouest [En ligne], 122-3 | 2015, mis en ligne le 30 octobre 2017, consulté le 22 avril 2019. URL : http://journals.openedition.org/abpo/3121 ; DOI : 10.4000/abpo.3121 


\title{
Le parlement de Bretagne et la réglementation du port d'armes $(1554-1789)$
}

\author{
Julien LE LEC \\ Étudiant en master 2 histoire - université Rennes 2
}

" Jamais l'abus des armes ne fut si grand ${ }^{1}$. " Tel est le constat désabusé que dresse le procureur général du roi au parlement de Bretagne dans un arrêt sur remontrance de 1610. Les guerres de la Ligue (1589-1598) avaient permis un armement massif de la population et le parlement s'engage dans cet arrêt à faire prévaloir l'interdiction du port d'armes afin d'obvier aux violences, vols et autres crimes. Sous l'Ancien Régime, les armes fascinent autant qu'elles sont craintes. Utilisées pour la chasse, lors de festivités, de conflits ou pour signifier une condition sociale, les armes sont omniprésentes dans la société et, de fait, préoccupent grandement les autorités qui, pour y remédier, bâtissent un arsenal législatif et réglementaire afin de prohiber le port d'armes.

Si l'historiographie s'est particulièrement intéressée à la période des $\mathrm{XIII}{ }^{\mathrm{e}}-\mathrm{XV}^{\mathrm{e}}$ siècles pour expliquer la mise en place de l'incrimination royale ${ }^{2}$, concernant l'Ancien Régime, aucune étude générale n'a été véritablement consacrée au port d'armes. La question est diluée dans une multitude d'ouvrages traitant de la police et l'ordre public ${ }^{3}$, la chasse ${ }^{4}$ ou les milices ${ }^{5}$.

1. Arch. dép. d'llle-et-Vilaine, 1Bf 229, 22 septembre 1610.

2. Voir particulièrement WENZ, Romain, Le port d'armes en France et la législation royale du milieu du XIII au milieu du XIV siècle, thèse de l'École des chartes, sous la direction de Claude Gauvard, Patrick Arabeyre, 2007. En introduction, l'auteur énumère les travaux portant sur cette question. Il a également publié “ "À armes notables et invasibles." Qu'est-ce qu'être armé dans le royaume de France à la fin du Moyen Âge? ", Revue historique, t. 3, n 671, 2014, p. 547-565.

3. Chagniot, Jean, Paris et l'armée au XVIII siècle. étude politique et sociale, Paris, Économica, 1985, p. 62-64; EL GHoul, Fayçal, La police parisienne dans la seconde moitié du XVIII siècle (1760-1785), dactyl., thèse d'histoire sous la direction de François Lebrun, université Rennes 2, 1993, vol. 3, p. 789-793; DENYS, Catherine, Police et sécurité au XVIII siècle dans les villes de la frontière franco-belge, Paris, L'Harmattan, 2002, p. 93-105.

4. Salvadori, Philippe, La chasse sous l'Ancien Régime, Paris, Fayard, 1996; Moriceau, Jean-Marc, L'homme contre le loup, Une guerre de deux mille ans, Paris, Fayard, 2011.

5. Descimon, Robert, " Milice bourgeoise et identité citadine à Paris au temps de la Ligue ", Annales, Économies, Sociétés, Civilisations, 48 année, n 4, 1993, p. 893-894. 
L'analyse a pu être approfondie par Christian Desplat dans un article sur l'armement de la population des Pyrénées occidentales lors des soulèvements ${ }^{6}$. En revanche, très peu de réflexions sont consacrées aux relations entre parlement et port d'armes, malgré des sources nombreuses. Henri Carré et Romain Bareau ont consacré quelques pages au cas breton mais sans approfondir le sujet ${ }^{7}$. Le travail pouvait être repris grâce aux arrêts qui ont été le point central de la réflexion. Les parlements disposent d'un pouvoir de police générale qui leur permet de promulguer des arrêts sur remontrance. Ceux-ci sont portés à la connaissance de tous par des cris publics et des affiches. Certains énoncent des prescriptions de portée générale (destinées à l'ensemble du ressort) ou territorialement limitées (à l'échelle d'une ville par exemple) et à caractère impersonnel : ils sont alors appelés arrêts de règlement.

Parmi les plus de 6000 arrêts sur remontrance promulgués par le parlement de Bretagne entre 1554 et 1789, 161 concernent le port d'armes. Ce corpus permet d'appréhender minutieusement l'encadrement d'une pratique courante, interdite majoritairement aux roturiers et autorisée aux nobles, officiers et militaires, même si les exemptions ne sont pas totales. Les règlements entendent pourfendre une violence considérée comme trop endémique. L'incrimination royale peut alors être interprétée comme une mesure d'ordre public qu'il faut assurer grâce à un appareil répressif. Étudier la réglementation du port d'armes invite plus largement à mener une réflexion, d'une part, sur la construction juridique et administrative du parlement, c'est-à-dire sur son pouvoir de police générale, et d'autre part, sur son affirmation comme puissance publique au sein de la province. Il importe ainsi de s'interroger sur la prérogative du parlement à réglementer le port d'armes en Bretagne par rapport aux autres institutions avant de mettre en lumière son affirmation. Ensuite, il convient d'examiner les enjeux de l'interdiction du port d'armes, du moyen de contrôle de la population à la question du maintien de l'ordre public. Enfin, il s'agira de se pencher de manière plus précise sur les armes condamnées par le parlement ${ }^{8}$.

6. Desplat, Christian, «Le peuple en armes dans les Pyrénées occidentales françaises à l'époque moderne ", dans Nicolas, Jean (dir.), Mouvements populaires et conscience sociale $X^{2} t^{e}-X I X X^{e}$ siècle, actes du colloque de Paris, 24-26 mai 1984, Paris, Maloine, 1985, p. 217-227. Voir également les travaux menés dans le cadre du programme ANR «Conflits et politisation " et en particulier le dossier n ${ }^{\circ} 118$ de 2011 des Annales de Bretagne et des Pays de l'Ouest, introduit par LAGADEC, Yann, "Prendre et/ou porter les armes entre les XIII ${ }^{\mathrm{e}}$ et XIX ${ }^{\mathrm{e}}$ siècles, un facteur de politisation? Quelques perspectives ", p. 7-20.

7. CARRÉ, Henri, Essai sur le Parlement de Bretagne après la Ligue (1598-1610), Paris, Quantin, 1888, p. 488-500; BAREAU, Romain, Les arrêts de règlement du parlement de Bretagne, thèse de droit, Rennes 1, 2000, p. 276-285.

8. Le propos présenté ici est tiré d'un mémoire de master (Les armes en Bretagne sous l'Ancien Régime. Étude menée à travers les arrêts sur remontrance du parlement de Bretagne (1554-1789), sous la direction de G. Aubert, université Rennes 2 juin 2015). Pour leur aide, remarques ou conseils lors de la rédaction de cet article, je tiens à remercier Gauthier Aubert, Mathieu Pichard et Pascale Gonzalez. 


\section{Quelle prérogative pour le parlement de Bretagne?}

Le réglementation sur le port d'armes se présente d'abord comme un relais du pouvoir royal. Par essence, l'objet de l'arrêt de règlement est de " compléter ou d'éclairer la loi du roi, voire de pallier son absence, mais en aucun cas il ne peut aller à l'encontre du texte législatif ${ }^{9}$ ". La question du port d'armes s'intègre dans ce moule car les parlementaires bretons reprennent en grande partie les 81 interdictions royales énoncées entre 1516 et 1785 . L'arsenal réglementaire sur le port d'armes est constitué de multiples références à la législation royale, souvent imprécises d'ailleurs. C'est le socle sur lequel la cour bâtit sa réglementation. Il s'agit surtout de rappeler l'existence des " edictz de sa maiesté ${ }^{10}$ ". Le 24 mai 1630, par exemple, on peut lire que "l'advocat general du roy entré en la cour a remonstré que par les edicts du roy, arrests et reglemens de la cour il est deffendu à toutes personnes de qualité non noble de porter armes à feu ${ }^{11}$ ". La juxtaposition dans la phrase entre " edicts du roy " et " arrests et reglemens de la cour " permet de rappeler l'existence et la force des arrêts du parlement, qui conservent une existence parallèle et complémentaire. Au-delà des seules références, les contenus des interdictions se ressemblent fortement : interdiction du port d'armes aux écoliers et domestiques, défense pour les roturiers de chasser et de porter une arme à feu, interdiction des armes dites " secrètes ". De même, la cour ajuste la sévérité des peines en fonction des évolutions des actes royaux, nous y reviendrons. $\mathrm{La}$ " coopération administrative " fonctionne donc pleinement ${ }^{12}$. Parfois, les arrêts se bornent à renouveler l'exécution des lois, par exemple en les citant intégralement. C'est ainsi le cas avec l'arrêt d'octobre $1700^{13}$ qui interdit le port de l'épée et des armes à feu à quiconque sauf aux nobles, officiers et ceux qui bénéficient d'un droit ou d'une charge pour en porter : il reprend l'ordonnance du mois précédent ${ }^{14}$.

À l'échelle de la province, d'autres institutions peuvent concurrencer le parlement. $\mathrm{Au} \mathrm{XVI}{ }^{\mathrm{e}}$ siècle, la cour ne semble avoir réglementé sur le port d'armes qu'à quatre reprises ${ }^{15}$. Certes, la perte de certains arrêts n'est pas à exclure, mais l'attention doit être portée sur le gouverneur de la province qui possède également dans la seconde moitié $\mathrm{du} \mathrm{XVI}^{\mathrm{e}}$ siècle une compétence pour réglementer le port d'armes. Dans les années 1560, le duc d'Étampes et son successeur en 1565, le vicomte de Martigues, édictent plu-

9. BAREAU, Romain, Les arrêts de règlement..., op. cit., p. 10.

10. Arch. dép. d'Ille-et-Vilaine, 1Bf 303, 11 mars 1622.

11. Arch. dép. d'Ille-et-Vilaine, 1Bf 1608, 24 mai 1630.

12. PAYEN, Philippe, Les arrêts de règlement du Parlement de Paris au XVIII siècle, Paris, PUF, 1997, p. 394.

13. Arch. dép. d'llle-et-Vilaine, 1Bf 1442, 6 octobre 1700.

14. ISAMBERT, Recueil général des anciennes lois françaises depuis 420 jusqu'à la Révolution, Paris, Belin-Le-Prieur, 1821-1833, t. XX, p. 369, ordonnance du 9 septembre 1700.

15. 3 août 1554 (Arch. dép. d'Ille-et-Vilaine, 1Bb 1), 21 octobre 1560 (Arch. dép. d'llleet-Vilaine, 1Bb 13), 12 juin 1589 (Arch. dép. d'Ille-et-Vilaine, 1Bf 63) et 14 août 1598 (Arch. dép. d'Ille-et-Vilaine, 1Bf 228). 
sieurs mesures de désarmement et d'interdiction du port d'armes, notamment dans les villes de Rennes et Nantes (1560, deux en 1563, 1565) ${ }^{16}$. La réglementation du port d'armes relève donc d'une compétence partagée entre la cour et les gouverneurs qui procède surtout d'une imprécision sur les pouvoirs de chacun concernant le port d'armes. Mais aux XVII ${ }^{\mathrm{e}}$ et XVIII ${ }^{\mathrm{e}}$ siècles, à l'échelle provinciale, les gouverneurs n'interviennent désormais plus dans ce domaine. Le port d'armes est réglementé par le parlement, tel un monopole qui relève pleinement de son pouvoir de police générale. Un double mouvement a donc lieu : captation par la cour de cette compétence dès le début du XVII ${ }^{\mathrm{e}}$ siècle et abandon de celle-ci par les gouverneurs. Seules quelques interventions d'autres autorités, mais sans conséquence, rognent cette prérogative.

$\mathrm{Au} \mathrm{XvIII{ } ^ { \mathrm { e } }}$ siècle, deux mesures de désarmement interviennent sans que la cour soit à l'origine de l'affaire. En 1738, le roi ordonne de faire désarmer les habitants de la forêt du duché de Quintin et des paroisses circonvoisines suite à la requête du duc de Lorges " parce qu'ils font un grand degast de gibier ${ }^{17}$ ". En réaction à l'ordonnance du 3 mars 1769 promulguée par le duc de Duras, commandant en chef en Bretagne, qui ordonne de faire désarmer les campagnes de la province ${ }^{18}$, l'avocat général du roi, Le Prestre de Châteaugiron est l'une des voix qui s'élèvent contre ce texte. Il pointe du doigt le problème de légitimité du duc de Duras à promulguer une telle ordonnance et réaffirme dans le même temps la compétence et l'autorité du parlement en matière de " police générale ", dont la réglementation des armes fait partie ${ }^{19}$. Ensuite, la critique de Le Prestre porte sur le contenu puisqu'il se révèle hostile au désarmement total de la population. Les habitants doivent pouvoir s'armer individuellement en vertu du droit d'autodéfense. La multiplicité des plaintes conduit le duc de Duras à atténuer la rigueur de son ordonnance le 27 mai. Il autorise que soient armés les habitants aisés et propriétaires. Ainsi, le parlement a pu considérer l'ordonnance du duc de Duras comme une atteinte à son autorité, à sa compétence en matière de police générale. Pourtant, malgré cela, rien n'inquiète véritablement le parlement, que ce soit l'intendant ou les municipalités.

L'arrivée d'un intendant en Bretagne en 1689 ne modifie en rien la prérogative parlementaire. Ceux qui se sont succédé n'ont, semble-t-il, jamais

16. RIVAULT, Antoine, Porter les armes. Institutions militaires, société civile, et affrontements religieux en Bretagne (vers 1550-1589), mémoire de master sous la direction de Philippe Hamon, École normale supérieure de Lyon, 2011, p. 231-233. Pour l'ordonnance de 1565, voir LA BORDERIE, Arthur de, "Documents sur l'histoire de Bretagne au XVI ${ }^{\mathrm{e}}$ siècle, tirés des archives impériales de Russie ", Bulletin de la Société archéologique et historique de Nantes et de Loire-Atlantique, 1877, t. 16, p. 54.

17. Arch. dép. d'Ille-et-Vilaine, C 155, lettre du comte de Saint-Florentin, 25 juillet 1738.

18. Le dossier est disponible sous la cote Arch. dép. d'Ille-et-Vilaine, C 156.

19. L'avocat général souligne le problème de concurrence; selon lui, c'est « le Parlement [qui est] chargé de la police générale de la Province ". Il fait notamment référence aux arrêts rendus en 1767, Arch. dép. d'Ille-et-Vilaine, C 156, lettre de l'avocat Le Prestre au duc de Duras, 16 avril 1769. 
cherché à obtenir une compétence pour réglementer le port d'armes ${ }^{20}$. De leur côté, par le biais des ordonnances et sentences de police, les municipalités participent au maintien du bon ordre en ville et la question du port d'armes fait partie de leurs prérogatives. Pour autant, elles demeurent subordonnées à la réglementation du parlement et à la législation royale. Une ordonnance municipale sur le port d'armes ne doit en aucun cas contredire les arrêts de la cour ni innover en matière de droit ${ }^{21}$. D'ailleurs, le procureur général possède un droit de regard important car, comme l'explique Philippe Payen, " nombre d'ordonnances sont prises à son instigation sans que, bien évidemment, les textes en portent trace ${ }^{22}$ ". Les ordonnances municipales rappellent également aux habitants les lois et arrêts. Le contenu et la rhétorique des interdictions sont souvent similaires comme en témoigne l'ordonnance de police de Brest de 1754 qui interdit " a tous clercs, facteurs et domestiques de porter épée, canne ou bâtons, soit de jour ou de nuit ny de porter des armes à feu et en tirer pendant la nuit ${ }^{23}$ " et fait référence à l'arrêt du 13 octobre 1744. Il ressort que les ordonnances de police remplissent une fonction d'actualisation et d'application des règlements ${ }^{24}$ et ne concurrencent pas le parlement dont la puissance réduit de fait l'envergure des municipalités de la province. À Rennes, le parlement s'ingère plus particulièrement dans les affaires de police urbaine, grâce aux arrêts de police générale de la cour, qui se présentent sous la forme d'ordonnances générales de police, traitant de plusieurs objets différents, dont le port d'armes. Des parlementaires, des commissaires de police et d'autres notables de la ville sont chargés d'organiser de rédiger ces arrêts. Dès lors, la police du parlement devient aussi la police de Rennes ${ }^{25}$. Un des signes de bonne entente entre la municipalité et le parlement est l'homologation de l'ordonnance, c'est-à-dire que la cour transforme en arrêt de règlement l'acte municipal. Le parlement lui confère alors une autorité plus grande et plus prestigieuse. Plusieurs concernent le port d'armes, comme en 1784 quand la cour promulgue deux arrêts sur homologation pour condamner l'usage, le commerce et le port d'armes par les jeunes de moins de 18 ans dans la ville de Rennes ${ }^{26}$. Preuve de leur subordination et du respect envers

20. La thèse de FrÉVILLE, Henri, L'intendance de Bretagne (1689-1790) : essai sur l'histoire d'une intendance en pays d'États au XVII' siècle, Rennes, Plihon, 1953 n'y fait aucune allusion. La même impression se dégage de la lecture de la récente thèse de Marie-ève Ouellet qui explique qu'en Bretagne, l'intervention réglementaire de l'intendant demeure limitée et n'intervient qu'après les autres institutions (OuEllet, Marie-ève, "Et ferez justice ". Le métier d'intendant au Canada et dans les généralités de Bretagne et de Tours au XVIII siècle (1700-1750), dactyl., thèse d'histoire, sous la direction de Thomas Wien et Philippe Hamon, universités de Montréal et Rennes 2, juillet 2014, p. 254).

21. PAYEN, Philippe, Les arrêts de règlement..., op. cit., p. 37-70.

22. Ibidem, p. 86.

23. CORRE, Armand, " Règlement de police pour la ville de Brest du mois de juin 1754 ", Bulletin de la Société Archéologique du Finistère, t. 21, 1894, p. 80-93.

24. PAYEN, Philippe, Les arrêts de règlement..., op. cit., p. 82.

25. BAREAU, Romain, Les arrêts de règlement..., op. cit., p. 30-32.

26. Arch. dép. d'Ille-et-Vilaine, 1Bf $1556^{2}, 30$ juin et 6 juillet 1784 . 
la réglementation du parlement, les deux ordonnances sont prises "sous le bon plaisir de la cour".

\section{L'affirmation par une production réglementaire importante : chronologie et géographie de l'interdiction du port d'armes}

Entre 1554 et 1789, le parlement édicte, on l'a dit, 161 arrêts qui interdisent le port d'armes. Entre 1640 et 1789, ils constituent 3,8 \% de l'ensemble des arrêts sur remontrance et $6,6 \%$ du nombre total d'arrêts de règlement. La fin des guerres de la Ligue constitue le moment essentiel où la cour entame une réglementation continue, jusqu'à la fin de l'Ancien Régime, sur le port d'armes. Par exemple, l'arrêt du 14 août 1598 s'inscrit dans le " pic " d'arrêts qui traduisent une volonté de pacifier la province après la promulgation de l'édit de Nantes. Il condamne les " laboureurs et aultres quy se licencient au preiudice de l'édict de la paix de porter harquebouzes, pystolles et aultres armes et tiennent encores nombre de parroisses barricadees contre la tranquilité et seureté qui doibt estre en ceste province ${ }^{27}$ ". Par conséquent, la cour interdit le port d'armes, l'édification de barricades et ordonne de surcroît le désarmement des laboureurs.

Ensuite, les arrêts sur le port d'armes connaissent une forte augmentation dans les années 1600-1640, non sans lien avec des difficultés qui se cumulent, telles que les crises épidémiques et les disettes qui provoquent des émeutes ${ }^{28}$, mais aussi les crises politiques (les affaires de Chalais et de La Rochelle). Des années 1640 au début des années 1760, les valeurs sont plus faibles et se stabilisent autour d'une moyenne d'un arrêt tous les deux ans. Cependant, la hausse est notable, avec douze arrêts entre 1675 et 1690, lorsque le parlement est déplacé à Vannes après la révolte du papier timbré, hausse constatée également pour l'ensemble des arrêts " ce qui pourrait être dû tant à un désir de se montrer zélé au service du roi après le choc de la révolte dite du Papier timbré qu'à la personnalité du nouveau premier président Ponchartrain ${ }^{29}$ ". Une autre période fructueuse en arrêts sur le port d'armes s'étale de 1766 à 1784 : 28 règlements sont prononcés. Cette recrudescence pourrait être une réponse à la hausse de la violence ${ }^{30}$, et/ ou provenir de la personnalité de l'avocat général du roi Le Prestre de

\footnotetext{
27. Arch. dép. d'Ille-et-Vilaine, 1Bf 228, 14 août 1598.

28. CROIX, Alain, La Bretagne aux XVI et XVII siècles : la vie, la mort, la foi, Paris, Maloine, 1980-1981, p. 253-339, 367-571.

29. AubERT, Gauthier, Hess, Aurélie, « Le parlement de Rennes était-il le parlement de Bretagne? Le témoignage des arrêts sur remontrances (XVI $-\mathrm{XVIII}^{\mathrm{e}}$ siècles) ", dans DAUCHY, Serge, Demars-Sion, Véronique, Leuwers, Hervé, Michel, Sabrina (dir.), Les Parlementaires acteurs de la vie provinciale XVIT-XVII $e^{e}$ siècles, Rennes, PUR, 2013, p. 163.

30. MEYER, Jean, La noblesse bretonne au XVIII siècle, Paris, EHESS, 1985 [1966], p. 1096. L'auteur note que de 1757 à 1786, la criminalité bretonne connaîtrait une augmentation "d'environ 50 \% pour les meurtres, et une multiplication par 7 des vols ", en lien avec l'augmentation des prix et la dégradation du niveau de vie, même s'il demeure prudent sur ces hypothèses, faisant remarquer que ces chiffres pourraient simplement traduire " une efficacité plus grande de la maréchaussée et des tribunaux répressifs ".
} 
Figure 1 - Répartition des arrêts sur remontrance interdisant le port d'armes par tranche de cinq ans (1554-1789)

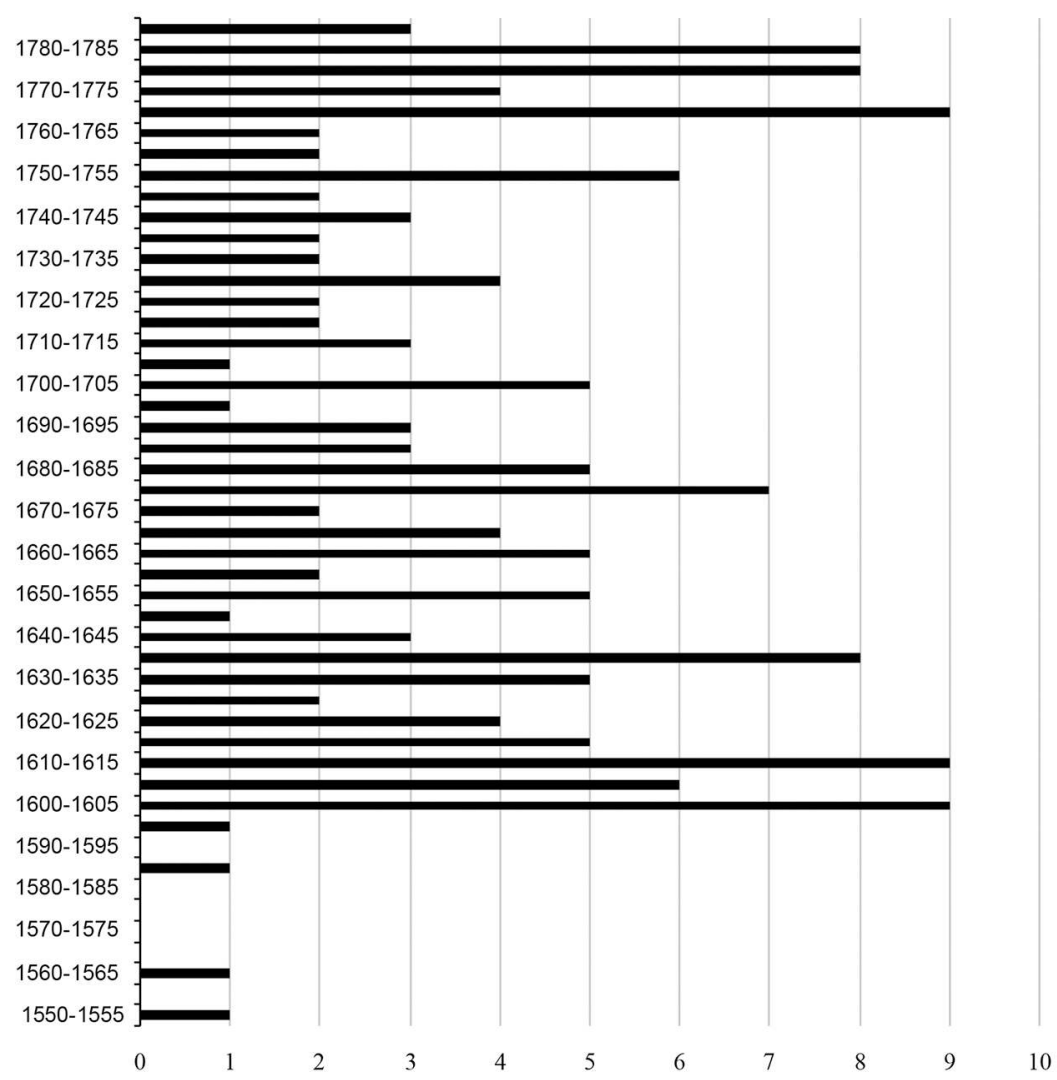

Châteaugiron. Son zèle se vérifie par la vitesse de réitération des arrêts car il en fait promulguer quatre en $1767^{31}$. Il est rare, en effet, que des arrêts qui se font référence se suivent aussi rapidement.

Pour mieux comprendre " le périmètre d'action " du parlement de Bretagne, Gauthier Aubert et Aurélie Hess invitent à regarder de plus près les différents thèmes ${ }^{32}$. Qu'en est-il des arrêts sur le port d'armes? Comment se répartissent-ils géographiquement?

Tous les arrêts ne peuvent être localisés : 39 arrêts (24,2 \%) ne ciblent pas une ou plusieurs paroisses précisément nommées ${ }^{33}$. Les arrêts généraux, qui concernent bien souvent la chasse, présentent une caractéris-

31. Arch. dép. d'Ille-et-Vilaine, 1Bf 1513, 31 janvier, 30 juin, 30 juillet et 10 décembre 1767.

32. Aubert, Gauthier, Hess, Aurélie, "Le parlement de Rennes... ", art. cit., p. 167.

33. Parmi eux, 35 sont des " arrêts généraux " qui couvrent l'ensemble du ressort. 

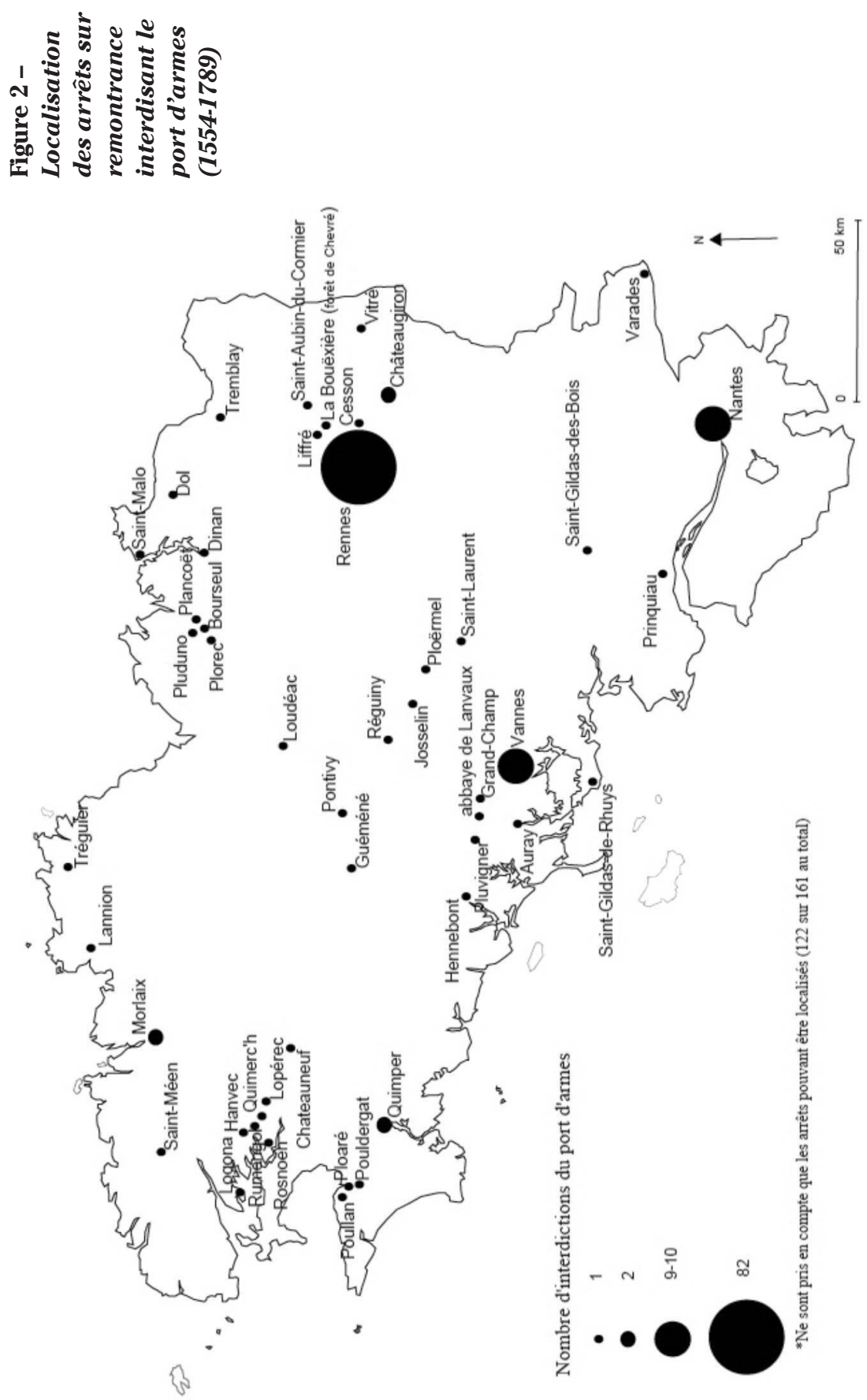
tique singulière : ils condamnent toujours le port des armes à feu (quelquefois associées aux épées, bâtons) mais jamais uniquement les armes blanches. Les autres arrêts précisent une ou plusieurs localisations. Les villes prennent une place importante, en particulier Rennes qui totalise 82 arrêts. En tant que capitale provinciale, siège du parlement et, en particulier au XVIII ${ }^{\mathrm{e}}$ siècle, des états, grande ville qui abonde en écoliers et domestiques, cette ville a toutes les raisons d'être surveillée de près par la cour. Les autres villes font pâle figure à côté : Nantes et Vannes comptent respectivement dix et neuf arrêts, et Quimper deux. L'importance des villes est déterminée aussi par une présence plus forte des sergents, des milices bourgeoises, qui surveillent la population, rédigent des procès-verbaux en cas de port d'armes illicite qui remontent ensuite au parlement. Dans les campagnes, les habitants sont plus éloignés des forces de l'ordre, les contrôles sont donc plus rares. La région de Vannes est la seconde région pour le nombre d'arrêts. Son importance tient au fait qu'entre 1675 et 1690, pendant que le parlement est à Vannes, la ville et ses environs ${ }^{34}$ concentrent l'activité réglementaire en matière de port d'armes, alors qu'aucun arrêt ne concerne Rennes. Que le parlement soit à Rennes ou à Vannes, c'est bien la ville où siège le parlement et la région environnante qui attirent l'attention des magistrats. Un autre groupe se dessine autour de Saint-Malo. Toutes les localisations à l'est de la presqu'île de Crozon ne concentrent qu'un seul arrêt interdisant aux roturiers de porter des fusils et de chasser ${ }^{35}$. Ainsi, le littoral prend une place importante. Les armes y sont très présentes à travers les milices gardes-côtes, les allées et venues de matelots qui ont pu servir comme corsaires, comme en 1748 où, à leur retour à Saint-Malo, après avoir servi dans les navires corsaires, beaucoup de jeunes gens " s'ingèrent de porter l'épée " dans la ville bien que " la liberté de la course soit cessée " obligeant la cour à rappeler alors l'interdiction du port de l'épée ${ }^{36}$.

\section{Les enjeux multiples d'une incrimination}

L'interdiction du port d'armes vise essentiellement à contrôler la population et à maintenir l'ordre public. Pour autant, toute la population n'y est pas astreinte. La défense de porter des armes concerne prioritairement les roturiers. Toutes les armes sont concernées, que ce soient les épées, les couteaux, les pistolets, les fusils, les bâtons ou les cannes. À Vitré, suite à des désordres qui se sont déroulés dans la ville, les "gents de basse condition " se voient interdire le port d'armes à feu, d'épées, de bâtons et de poignards ${ }^{37}$. Ces interdictions qui s'adressent au monde de la roture se rencontrent aussi quand le parlement condamne la chasse qui est réservée aux seigneurs. Dans 23 arrêts, il interdit régulièrement aux paysans de

34. Par exemple, deux arrêts condamnent le port d'armes durant la chasse sur l'île de Rhuys (Arch. dép. d'Ille-et-Vilaine, 1Bf 1440, 16 juillet 1683 et 13 juillet 1684).

35. Arch. dép. d'Ille-et-Vilaine, 1Bf 1596, 29 février 1772.

36. Arch. dép. d'Ille-et-Vilaine, 1Bb 697, 9 septembre 1748.

37. Arch. dép. d'Ille-et-Vilaine, 1Bf 1223, 6 août 1718. 
porter des armes à feu (escopettes, arquebuses, fusils) et plus rarement d'autres armes telles que les arbalètes ${ }^{38}$. En ville, outre les interdictions qui s'adressent à toute la population, le parlement formule ses réprimandes contre certaines catégories sociales, jugées plus dangereuses que d'autres : ce sont les domestiques, écoliers, clercs, artisans, gens de boutique, etc.; 74 arrêts les concernent. En tant que roturiers, ils ne peuvent porter aucune arme. Dans les condamnations, les armes blanches sont les plus mentionnées (45,9\%), avant les cannes et bâtons (36,2\%), et enfin les armes à feu (14,1 \%). La canne et le bâton représentent l'arme par excellence des couches populaires, surtout au XVIII siècle. Tous ces individus sont attachés au port d'armes au point qu'il devient une revendication courante, ce qui rend d'autant plus difficile l'application des arrêts. En 1789, les cahiers de doléances se partagent entre ceux qui veulent que le port d'armes soit interdit à tous à l'exception des militaires ${ }^{39}$ et ceux qui veulent que les roturiers puissent porter des armes ${ }^{40}$.

Jusqu'au premier quart du XVIII ${ }^{\mathrm{e}}$ siècle, seuls les écoliers, domestiques, clercs et cochers sont mentionnés. Puis sont inclus les porteurs de chaises, facteurs, garçons de boutique, artisans, perruquiers, etc. Un arrêt de 1778 est révélateur de leurs désordres provoqués par le port d'armes :

"L'abus du port des armes et de batons, de tout tems proscrit par les ordonnances, arrêts et règlemens de la cour, s'est renouvellé dans cette ville de Rennes : l'usage des cannes est surtout devenu général, les étudiants en droit, les clercs, les facteurs, les jeunes gens de touttes les classes, arts et metiers en portent le jour et la nuit. La licence est telle qu'ils osent en porter jusques dans le palais de la justice. Il y a même de ces cannes qui renferment des epées ou des lances, ce qui donne matière a des disputes et à des batteries frequentes. Ces desordres sont egalement préjudiciables au bon ordre et à la sureté publique ${ }^{41}$. "

Parmi ces catégories, les domestiques et les écoliers sont le plus souvent condamnés. Afin de maintenir la "tranquillité publique", la police des domestiques use de diverses mesures, et l'interdiction du port d'armes est le plus souvent rappelé ${ }^{42}$. Le parlement la réitère dans 55 règlements (34,2 \% des 161 arrêts). La présence pléthorique des laquais à Rennes trouve un écho dans les nombreux conflits armés. La domesticité est perçue comme une classe turbulente et agitée, ce qui justifie l'abondante réglementation qui tend à les encadrer. Il est surtout reproché aux domestiques

38. Elles sont mentionnées seulement dans les arrêts de la première moitié du XVII ${ }^{\mathrm{e}}$ siècle.

39. IsBLED, Bruno, "Le cahier de doléances de la sénéchaussée de Fougères ", Mémoires de la Société d'Histoire et d'Archéologie de Bretagne, t. 74, 1996, p. 5-44.

40. Thomas-Lacroix, Pierre, "Les cahiers de doléances de la sénéchaussée d'Hennebont ", Mémoires de la Société d'Histoire et d'Archéologie de Bretagne, t. 35, 1955, p. 52-103; SAVINA, Jean et BERnARD, Daniel, Cahiers de doléances des sénéchaussées de Quimper et de Concarneau pour les États généraux de 1789, Rennes, 1927.

41. Arch. dép. d'Ille-et-Vilaine, 1Bb 728, 12 août 1778.

42. Gutton, Jean-Pierre, Domestiques et serviteurs dans la France de l'Ancien Régime, Paris, Aubier-Montaigne, 1981, p. 139; PETITFRÈRE, Claude, L'œil du maître. Maîtres et serviteurs, de l'époque classique au romantisme, Paris, Éditions Complexe, 1986, p. 180. 
de porter des épées, des bâtons et des cannes. D'ailleurs, leurs maîtres sont tenus pour responsables de la conduite de leurs domestiques en les empêchant de porter des armes ou même leur propre épée. Les domestiques participent d'" une double violence " : celle des milieux populaires d'où ils sont originaires, mais aussi celle de leurs maîtres ${ }^{43}$. En portant l'épée, le domestique agit par "mimétisme social ", comme une imitation du modèle nobiliaire, celui de son maître surtout. Avoir l'épée au côté offre un éclat d'honneur supplémentaire.

\section{Tableau 1 - Nombre d'apparitions des différentes catégories sociales} dans les 74 arrêts où elles sont mentionnées ${ }^{44}$

\begin{tabular}{|l|c|c|c|c|c|}
\hline & $1600-1650$ & $1650-1700$ & $1700-1750$ & $>1750$ & Total (\%) \\
\hline domestiques* $^{*}$ & 17 & 14 & 10 & 14 & $55(33,1 \%)$ \\
\hline écoliers & 15 & 12 & 13 & 11 & $51(30,7 \%)$ \\
\hline clercs & 2 & 4 & 8 & 9 & $23(13,9 \%)$ \\
\hline facteurs & & & 4 & 11 & $15(9,0 \%)$ \\
\hline garçons de boutique & & & 1 & 9 & $10(6,0 \%)$ \\
\hline cochers & 2 & 1 & & & $3(1,8 \%)$ \\
\hline artisans & & & & 2 & $2(1,2 \%)$ \\
\hline manœuvriers & & & 1 & & $1(0,6 \%)$ \\
\hline apprentis & & & & 1 & $1(0,6 \%)$ \\
\hline marchands & & & & 1 & $1(0,6 \%)$ \\
\hline ouvriers & & & 1 & & $1(0,6 \%)$ \\
\hline perruquiers & & & 1 & & $1(0,6 \%)$ \\
\hline porteurs de chaise & 1 & & & & $1(0,6 \%)$ \\
\hline marginaux * & 37 & 31 & 39 & 59 & $166(100,0 \%)$ \\
\hline Total & & & &
\end{tabular}

* Englobe les laquais, domestiques, gens de livrée, pages et valets (de chambre).

** L'arrêt évoque les " vacabons, faineans, gens desbauchez, sans adveu et inutilz ".

Quant aux écoliers, le nombre d'interdictions du port d'armes qui les concernent s'élève à 51 (la première date de $1607^{45}$ ), dont 17 où ils sont les seuls à faire l'objet de la condamnation. Les écoliers manient autant l'épée que la plume puisqu'ils sont particulièrement réputés pour être insolents et violents ${ }^{46}$, n'hésitant pas à s'engager dans des rixes et à faire parler leur

43. GutTon, Jean-Pierre, Domestiques et serviteurs..., op. cit., p. 139.

44. Souvent, un arrêt mentionne plusieurs de ces catégories urbaines, ce qui explique le nombre de 166.

45. Arch. dép. d'Ille-et-Vilaine, 1Bb 109, 30 août 1607.

46. Cassagnes-Brouquet, Sophie, La violence des étudiants au Moyen Âge, Rennes, OuestFrance, 2012; Idem, "La violence des étudiants à Toulouse à la fin du XV et au XVI siècle (1460-1610) ", Annales du Midi, vol. 94, n 158, 1982, p. 245-263; BRoKLISS, Laurence, 
sentiment de contestation. Ces violences permettent de mieux considérer la place des écoliers au sein des sociétés urbaines. Le port d'armes est donc un prisme intéressant pour étudier cette population scolaire et universitaire. En 1665, « il se faict tous les jours [à Rennes] assemblee de plusieurs escolliers, lesquels attaquent, battent et excedent tous les gens de livrees armez d'epees, pistollets et bastons, attaquent mesme les carosses et battent les vallets " ${ }^{47}$. Le 29 juin 1722, la rue Saint-Germain à Rennes devient le théâtre d'une querelle entre écoliers et artisans " s'estant armés les uns et les autres de sabres, épées, bâtons et pistolets ${ }^{48}$ ". Dans chaque cas, la cour leur rappelle l'interdiction du port d'armes. Si les arrêts ne livrent pas de détails sur les épées utilisées, il est possible de se référer aux cas des autres universités où les écoliers utilisent aux XVI ${ }^{\mathrm{e}}$ et début du XVII ${ }^{\mathrm{e}}$ siècle l'épée à deux mains ${ }^{49}$. Lourde, difficilement maniable, elle sera remplacée par la rapière. L'arsenal réglementaire confirme l'image de l'écolier armé, l'épée au côté. En 1637, «il se commet un tres grand desordre dans la ville et forbourgs de Vennes par la licence que ont pris les escoliers quy estudient sous les peres jesuites dudit Vennes de porter des espees ${ }^{50}$ ". Le constat est le même un siècle plus tard : la cour déplore " la facilité que l'on a de laisser porter l'épée aux ecoliers de droit, a ceux du college et aux clercs de Nantes ${ }^{51}$ ". Cette arme est la plus portée, en tout cas la plus condamnée. Au-delà de l'interdiction du port d'armes, la cour ordonne, à cause du danger qu'ils font peser sur le bon ordre social, à douze reprises durant les deux premiers tiers du XVII ${ }^{\mathrm{e}}$ siècle, de désarmer les écoliers, c'est-à-dire de leur interdire toute possession d'armes dans leurs maisons ou pensions. De fait, les hôtes qui logent les écoliers ont un rôle théorique important car ils ont le devoir de se saisir de leurs armes et d'empêcher qu'ils sortent armés dans les rues. L'encadrement de la population roturière, même s'il est peu efficace, se veut donc rigoureux afin de maintenir le bon ordre.

Réglementer le port d'armes relève du travail de police, considéré sous l'Ancien Régime comme synonyme d' "administration ». Fondée sur l'idée du maintien d'une organisation sociale garante du bon ordre public, la police englobe des domaines variés concernant la bonne gestion urbaine, dont la réglementation du port d'armes qui traduit donc un enjeu essentiel : celui du maintien de l'ordre public. Depuis Nicolas Delamare ${ }^{52}$, la police se divise

"Contenir et prévenir la violence. La discipline scolaire et universitaire sous l'Ancien Régime (XVII ${ }^{\mathrm{e}}$-XVIII ${ }^{\mathrm{e}}$ siècles) ", Histoire de l'éducation, $\mathrm{n}^{\circ}$ 118, 2008, p. 51-66.

47. Arch. dép. d'Ille-et-Vilaine, 1Bf 847, 15 juin 1665.

48. Arch. dép. d'Ille-et-Vilaine, 1Bf 1443, 3 juillet 1722.

49. Brioist, Pascal, Drévillon, Hervé, SERnA, Pierre, Croiser le fer. Violence et culture de l'épée dans la France moderne (XVI -XVIII ${ }^{e}$ siècle), Paris, Seyssel, Champ Vallon, coll. "Époques ", 2002, p. 24, 47; Dans Pantagruel, Rabelais écrit : " De là vint à Toulouse où apprint fort bien à danser et à jouer de l'espée à deux mains comme est l'usance des escholiers en ladicte Université ", cité par LEGUAY, Jean-Pierre, La rue au Moyen Âge, Rennes, Ouest-France, 1984, p. 160.

50. Arch. dép. d'Ille-et-Vilaine, 1Bf 484, 9 mai 1637.

51. Arch. dép. d'Ille-et-Vilaine, 1Bf 1445, 18 novembre 1734.

52. Delamare, Nicolas, Traité de la police, Paris, 1722, préface. 
traditionnellement en onze chapitres principaux. Le sixième, la "sûreté publique ", considérée comme "l'une des plus importantes matieres de la police ${ }^{53}$ ", englobe la question du port d'armes. Depuis, tant chez les auteurs du XVII siècle que chez les historiens, le port d'armes est demeuré dans le domaine de la "sûreté publique ". À plusieurs reprises dans la réglementation parlementaire, des expressions telles que "repos public ", " ordre public ", " sûreté publique ", " liberté publique " sont utilisées pour donner du poids à la prohibition. L'étude des arrêts de police générale de la cour permet de mettre en perspective la question du port d'armes par rapport au domaine plus vaste qu'est la sûreté publique. Ces arrêts prennent des dispositions sur des questions diverses de police urbaine. Sous la forme d'un court article (3-4 lignes), l'interdiction du port d'armes est rappelée ponctuellement, à 27 reprises (7,9 \%) sur les 340 arrêts dénombrés entre 1676 et 1789 . Ils cernent tout particulièrement les catégories urbaines (écoliers, clercs, laquais et autres) et confirment l'omniprésence du triptyque épée, canne, bâton, qui sont véritablement les armes de la ville. Lorsqu'un article est consacré au port d'armes, c'est en lien avec la tenue des états de Bretagne, si bien que l'enjeu d'ordre public prend tout son sens car il s'agit de contrôler la nombreuse domesticité qui accompagne les nobles venus spécialement à Rennes pour la participation aux États et qui sont d'ailleurs de plus en plus nombreux au cours de l'Ancien Régime $^{54}$.

La sécurité nocturne constitue un cas particulièrement intéressant de la production réglementaire sur le port d'armes pour faire prévaloir l'enjeu de l'ordre public ${ }^{55}$. Les violences et tapages causés par les " coureurs de nuit " (souvent des écoliers, domestiques, soldats) conduisent les autorités à prendre diverses mesures afin de " policer " un moment qui leur échappe en partie. Alors que c'est sous Louis XIV que la nuit prend une importance singulière dans la législation royale sur le port d'armes ${ }^{56}$, l'attention est plus précoce (dès le début du XVII ${ }^{\mathrm{e}}$ siècle) dans la réglementation du parlement. Au total, ce sont 59 arrêts qui font mention de la nuit. Tous les arrêts consacrés spécifiquement au moment nocturne défendent à quiconque de " vaquer " dans les rues avec des armes. Les nobles sont également concernés par cette interdiction mais peuvent porter leur épée la nuit seulement s'ils sont éclairés. En revanche, ils ont interdiction de porter des armes à feu.

Ainsi, l'interdiction du port d'armes qui se répand dans les villes ne vise pas à désarmer les habitants mais seulement à restreindre la violence quo-

53. Ibidem.

54. MEYER, Jean, La noblesse bretonne..., op. cit., p. 77.

55. Cabantous, Alain, Histoire de la nuit (XVII - -XVIII ${ }^{e}$ siècles), Paris, Fayard, 2009; MuCHEMBLED, Robert, "La violence et la nuit sous l'Ancien Régime ", Ethnologie française, t. XXI, n 3, 1991, p. 237-242. LEMAîTRE, Alain-Jean, "L'illumination publique et la sécurité à Rennes au XVIII ${ }^{\mathrm{e}}$ siècle ", Mémoires de la Société d'Histoire et d'Archéologie de Bretagne, t. 84, 2007, p. 187-210.

56. Voir les déclarations royales de décembre 1660 et 1666. 
tidienne. Elle est notamment un moyen pour les autorités de " discipliner la violence ${ }^{57}$ ". La législation répétée sur le port d'armes permet de définir une " violence publique, celle qui enfreint l'ordre public ${ }^{58}$ ". Le but de la prohibition est surtout d'empêcher une violence potentielle, celle qui n'est pas encore advenue car le contrevenant est arrêté " pour s'être donné les moyens d'une violence éventuelle ${ }^{59}$ ". Afin de veiller à sa bonne application, l'incrimination royale du port d'armes est encadrée par un appareil répressif qui a sensiblement évolué, à l'exception de la confiscation de l'arme. Celui-ci se divise en trois phases depuis le XIII ${ }^{\mathrm{e}}$ siècle jusqu'au XVIII ${ }^{\mathrm{e}}$ siècle. Au Moyen Âge, il est caractérisé par une tarification fixe (60 sous pour les roturiers, 60 livres pour les nobles ${ }^{60}$ ) avant que la répression ne devienne plus sévère $\mathrm{au} \mathrm{XVI}^{\mathrm{e}}$ et dans la première moitié du XVII ${ }^{\mathrm{e}}$ siècle avec une prédominance de la peine de mort. Cette dernière cesse de s'appliquer en fonction de la condition sociale des individus, le curseur de sévérité étant désormais basé sur d'autres critères comme le type d'armes et le fait d'être en groupe. À partir du règne de Louis XIV et jusqu'à la fin du XVIII ${ }^{\mathrm{e}}$ siècle, le port d'armes est à nouveau puni majoritairement par des amendes et de la prison. Prenant acte de la législation royale, le parlement prévoit majoritairement la peine de mort dans les arrêts qui interdisent le port d'armes à feu (il peut y avoir aussi des armes blanches) à toutes personnes qui mêlent port d'armes et attroupement. Les armes à feu sont considérées comme plus dangereuses que les armes blanches, ce qui justifie une répression plus sévère. Un arrêt de février 1623 distingue la peine selon le type d'armes : "Deffenses a touttes personnes de quelque quallitté et condittion qu'elles soient de porter pistolletz et aultres armes a feu en cette ville et forbourgs de jour ou de nuict sur paine de la vye et a touttes personnes n'estans de quallitté noble de porter espee et pongnardz sur paine de punition corporelle ${ }^{61}$. " La dernière peine de mort est mentionnée dans un arrêt de $1675^{62}$, dans un contexte de révolte ce qui explique la sévérité. Après 1675 , ce sont les amendes (entre 50 et 100 livres) et les peines de prison qui sont récurrentes, au moment où le pouvoir royal adoucit les peines. Concernant la chasse, la peine est tarifaire. Les sommes varient durant la première moitié du XVII ${ }^{\mathrm{e}}$ siècle, avant de se fixer à 500 livres par la suite. Pour les écoliers, domestiques, clercs, etc., deux pratiques punitives se succèdent : au XVII ${ }^{\mathrm{e}}$ siècle, prévalent les peines corporelles; au XVIII ${ }^{\mathrm{e}}$ siècle, la condamnation à la prison. Le parlement use donc de peines différentes selon les motifs de l'arrêt et les armes visées.

57. GAUVARD, Claude, "Discipliner la violence dans le royaume de France aux XIV et $\mathrm{XV}^{\mathrm{e}}$ siècles : une affaire d'état? ", dans Disziplinierung im Alltag des Mittelalters und der frühen Neuzeit, Vienne, 1999, p. 178.

58. GAUVARD, Claude, " Violence licite et violence illicite dans le royaume de France à la fin du Moyen Age ", dans Memoria y civilización, t. 2, 1999, p. 87-105.

59. WenZ, Romain, Le port d'armes en France..., op. cit., p. 181.

60. Ibidem, p. 238-250.

61. Arch. dép. d'Ille-et-Vilaine, 1Bf 303, 10 février 1623.

62. Arch. dép. d'Ille-et-Vilaine, 1Bf 849, 18 juillet 1675. 
Les interdictions du port d'armes sont compensées par des exceptions accordées à certains groupes d'individus. D'abord ce sont les nobles, officiers et militaires qui bénéficient d'exemptions. Le port d'armes devient dès lors un privilège, un signe de distinction sociale. Ensuite, ce sont les autres roturiers qui sont autorisés de manière temporaire à porter des armes. Les exceptions se fondent sur trois critères : l'appartenance à un ordre privilégié, l'exercice d'une fonction particulière et la circonstance " exceptionnelle " (les voyageurs) ${ }^{63}$. Le privilège du port d'armes pour les nobles, officiers royaux et militaires n'est pas total, il doit être nuancé en fonction du type d'armes (armes blanche ou armes à feu), des moments (jour/nuit) et des lieux (le palais par exemple) où ces armes sont portées. Nobles, militaires et officiers sont autorisés à porter des armes blanches. Le privilège du port de l'épée par la noblesse ne repose sur aucun texte normatif, mais relève plutôt d'un usage qui a fini par être entériné par la législation ${ }^{64}$. À plusieurs reprises, le parlement confirme cette distinction honorifique, comme en 1631 où il autorise les nobles à " porter leurs espées au costé ${ }^{65}$ ». En 1767, l'avocat général Le Prestre écrit que le port de l'épée est une "distinction privative aux nobles, aux militaires et aux officiers brevetés du roi ${ }^{66}$ ". Certains lieux résistent au privilège, tels les tribunaux et notamment le parlement où il est interdit de porter l'épée ${ }^{67}$. En 1554, quelques mois après la création du parlement, le duc d'Étampes, gouverneur de Bretagne, entend siéger tout en portant l'épée, ce que refusent les parlementaires ${ }^{68}$. Concernant les armes à feu, le parlement interdit à la noblesse d'en porter en dehors de la chasse avant le règne de Louis XIV. En 1630, les nobles sont explicitement mentionnés dans l'interdiction :

" Enjoint a tous gentilzhommes faisant profession des armes estans en cette ville de Rennes de porter leurs espees sans les bailler a leurs laquais et autres suivantz, leur fait deffances de porter pistolletz et aultres armes a feu en cettedite ville et fauxbourgs ${ }^{69}$."

À partir du règne de Louis XIV, la cour applique la législation royale qui autorise désormais les " gentilzhommes faisant profession des armes " à porter des armes à feu, mais cela ne concerne qu'une partie de la noblesse.

63. RenAult, Marie-Hélène, " Le port d'armes, de l'épée à la bombe lacrymogène », Revue de science criminelle, $\mathrm{n}^{\circ}$ 3, Dalloz, 1999, p. 523.

64. Wenz, Romain, Le port d'armes en France..., op. cit., p. 204.

65. Arch. dép. d'Ille-et-Vilaine, 1Bf 482, 14 mars 1631.

66. Arch. dép. d'Ille-et-Vilaine, 1Bf 1513, 10 décembre 1767.

67. Arch. dép. d'Ille-et-Vilaine, 1Bf 849, 21 novembre 1672 : " Faict deffences a touttes personnes de qualité et aultres d'entrer ny se promener dans les galleries du pallais avec leurs espees. "

68. Arch. dép. Ille-et-Vilaine, 1Bb 1, 13 août 1554. Le port de l'épée par les gouverneurs est analysé dans l'article de RIVAULT, Antoine, " "Monsieur le gouverneur est entré en la cour". Les entrées des gouverneurs de province au parlement de Bretagne pendant les guerres de religion (1554-1598) " publié dans ce même dossier.

69. Arch. dép. d'Ille-et-Vilaine, 1Bf 482, 26 avril 1630. 
Les employés des fermes ${ }^{70}$ ou plusieurs officiers comme ceux des eaux et forêts ${ }^{71}$ peuvent aussi porter des armes à feu.

Pour le reste des roturiers, les autorisations de port d'armes ne sont que temporaires et ponctuelles, mis à part pour les voyageurs qui peuvent porter des armes dès qu'ils sont sur les routes ${ }^{72}$. Les dérogations s'inscrivent dans trois domaines : la chasse aux loups, l'arrestation des malfaiteurs et les prises d'armes dans le cadre des levées de communes. Face aux ravages que causent les loups dans le royaume, les autorités choisissent d'organiser des battues. L'augmentation du nombre des prédateurs à la fin du $\mathrm{XVI}^{\mathrm{e}}$ et au début du XVII ${ }^{\mathrm{e}}$ siècle en Bretagne ${ }^{73}$ conduit le parlement à organiser des battues à trois reprises (en $1599^{74}, 1600^{75}$ et $1611^{76}$ ). Les paysans peuvent donc s'armer sous le contrôle de la noblesse locale. Mais à aucun moment, contrairement au pouvoir royal et à d'autres parlements, la cour n'autorise explicitement l'utilisation d'armes à feu ${ }^{77}$. En 1599, elle rature le terme " en armes ", signe d'une crainte qui se confirme dans les arrêts suivants. En 1611 , les paysans peuvent seulement s'équiper de " bastons ferrez " pour chasser le loup. La dernière battue, organisée en 1743, autorise que les paysans soient " armés $^{78}$ ", là encore sans plus de précision. Les habitants peuvent également prendre les armes pour aider la patrouille à arrêter des malfaiteurs. En 1634, afin d'empêcher que les domestiques ne vaguent dans les rues l'épée à la main, les sergents et huissiers doivent les faire arrêter. La cour demande également " à tous habitans de ladite ville en cas de rebellion de les secourir et assister avec halebardes, espées, pertuizaines ou autres bastons ferrez lorsqu'il seront apellez au secours ${ }^{79}$ ". En 1695, " en cas de revolte, [la cour] enjoint a tous habitants du quartier de sortir armés au moindre bruit ${ }^{80}$ ". Enfin, plusieurs arrêts datant des guerres de la Ligue et du début du XVII ${ }^{\mathrm{e}}$ siècle autorisent les habitants des paroisses à prendre les armes contre des ennemis : ce sont les levées de communes. En Bretagne, dans les années 1621-1622, les craintes de débarquements des troupes huguenotes incitent le parlement à s'y préparer. Fin septembre 1622, il

70. Ordonnance du 21 août 1681 concernant le droit des fermes, Titre commun, art. XI.

71. Ordonnance sur les eaux et forêts d'août 1669, Titre XXX. L'autorisation est reprise dans un arrêt du 30 mars 1754 (Arch. dép. Ille-et-Vilaine, 1Ba 69).

72. Voir les déclarations royales de 1660, 1679 et 1700. Le parlement, dans l'application de l'ordonnance de 1700 n'en fait pas pas mention. Seul l'arrêt du 31 janvier 1757 (Arch. dép. d'Ille-et-Vilaine, 1Ba 69) l'évoque.

73. MORICEAU, Jean-Marc, L'homme contre le loup. Une guerre de deux mille ans, Paris, Fayard, 2011, p. 82-88; Chauvin, Monique, Lagadec, Yann, Pennec, Jos, Rannou, Yves, " Le loup et l'homme dans le cadre de l'actuel département d'Ille-et-Vilaine (XVI ${ }^{\mathrm{e}}$-XIX ${ }^{\mathrm{e}}$ siècle) ", Bulletin et mémoires de la Société archéologique et historique d'Ille-et-Vilaine, 2009, p. 239-266.

74. Arch. dép. d'Ille-et-Vilaine, 1 Bf 228, 12 janvier 1599.

75. Arch. dép. d'Ille-et-Vilaine, 1 Bf 228, 14 octobre 1600.

76. Arch. dép. d'Ille-et-Vilaine, 1 Bf 229, 30 mars 1611.

77. C'est le cas par exemple du parlement de Dole en 1598, MoricEAU, Jean-Marc, L'homme contre le loup..., op. cit., p. 172.

78. Arch. dép. d'Ille-et-Vilaine, 1 Bf 1590, 17 septembre 1743.

79. Arch. dép. d'Ille-et-Vilaine, 1 Bf 483, 24 mars 1634.

80. Arch. dép. d'Ille-et-Vilaine, 1Bh 2, 2 décembre 1695. 
donne l'ordre " a tous les subiectz de sa majesté des parroisses voisines des costes de la mer de s'assambler avecq leurs armes [...] pour s'opposer aux dessains et incursions que pourroient faire les rebelles et ennemys de sa maiesté ${ }^{81}$ ". Dans le cadre de la défense rurale ${ }^{82}$, le parlement demande donc aux populations littorales de se tenir prêtes à combattre avec leurs propres armes pour défendre la sûreté de la province.

\section{Les armes condamnées}

L'expression " port d'armes " et les adjectifs qui qualifient les armes sont l'héritage d'un lexique juridique utilisé par la monarchie et le parlement de Paris du XIII ${ }^{\mathrm{e}}$ au XV $\mathrm{XV}^{\mathrm{e}}$ siècle ${ }^{83}$, héritage qui a connu depuis des évolutions. Dérivée de l'expression latine portatio armorum, la formule " port d'armes " s'est diffusée à partir du XIV ${ }^{\mathrm{e}}$ siècle au moment même où l'incrimination se met en place. Si l'expression est couramment utilisée à la fin du Moyen Âge et préférée à l'énumération de listes d'armes, la situation s'inverse entre le $\mathrm{XVI}^{\mathrm{e}}$ et le $\mathrm{XVIII}{ }^{\mathrm{e}}$ siècle : les ordonnances royales et les arrêts du parlement citent en moyenne entre deux et quatre armes et n'utilisent que rarement l'expression " port d'armes " dans les interdictions. Le parlement de Bretagne use de différents adjectifs pour qualifier les armes qu'il est interdit de porter : elles sont dites " offensives " (15 fois), " invasibles " (1), " prohibées " (6) ou " défendues " (8). Ce sont principalement l'ensemble des armes à feu (arquebuses, escopettes, mousquets, fusils, carabines, pistolets), les armes de jet (arbalètes), d'hast (lances, hallebardes), les épées et toutes sortes de couteaux, poignards, coutelas, et les bâtons et cannes. L'utilisation courante de ce lexique par la royauté et les différents parlements montre que les magistrats usent d'un vocabulaire juridique commun hérité, de la période médiévale et qui a perduré sous l'Ancien Régime. Cette culture juridique a normalisé les interdictions du port d'armes, y compris dans les ordonnances municipales.

En plus d'une approche lexicale, l'étude de la réglementation du port d'armes permet une mise en perspective des différentes armes mentionnées par le parlement puisque leur évolution technique (développement des armes à feu, multiplication des armes " cachées ") nourrit la réglementation au fil des années. De 1554 à 1789, pour l'ensemble des arrêts, les armes à feu (34,2\%) et les armes blanches (34,7 \%) sont les deux catégo-

81. Arch. dép. d'Ille-et-Vilaine, 1Bb 139, 28 septembre 1622.

82. Sur les levées de communes et défenses rurales, voir les travaux de Philippe Hamon, notamment " "Aux armes paysans!" : les engagements militaires des ruraux en Bretagne de la fin du Moyen Âge à la Révolution ", Mémoires de la Société d'histoire et d'archéologie de Bretagne, t. XCII, 2014, p. 221-244; Idem, "Pourquoi nous combattons. Réflexions sur l'engagement des communautés rurales dans les guerres de la Ligue ", dans DAUBRESSE, Sylvie, HAAN, Bertrand (dir.), La ligue et ses frontières. Engagements catholiques à distance du radicalisme à la fin des guerres de religion, Rennes, PUR, 2015, p. 95-110.

83. Une étude lexicale de l'interdiction du port d'armes au Moyen Âge est présentée dans Wenz, Romain, Le port d'armes en France..., op. cit., p. 193-210. 
ries d'armes les plus souvent interdites, devant les bâtons et les cannes (21,5 \%). Les armes à traits (arbalètes), les armures, et les canons ne représentent que $2 \%$ des armes interdites. Avec les armes de guerre (piques, hallebardes), elles tombent rapidement en désuétude. Au XVII ${ }^{\mathrm{e}}$ siècle, ce sont les armes à feu qui sont le plus souvent interdites, suivies des armes blanches avant qu'au siècle suivant, les règlements s'attachent plus à prohiber les armes blanches et surtout les cannes et bâtons. Ainsi, les arrêts du XVIII ${ }^{\mathrm{e}}$ siècle mentionnent fréquemment les "épées, cannes, bâtons ".

L'épée est de loin l'arme la plus citée (79 fois, soit 22,3\% de l'ensemble des mentions d'armes et $64,2 \%$ des armes blanches). Alors que les armes à feu sont toujours associées à l'idée de danger, rares sont les arrêts qui traitent du caractère dangereux de l'épée. Les parlementaires se concentrent surtout sur les personnes autorisées ou non à la porter. $\mathrm{Au}$ XVIII ${ }^{\mathrm{e}}$ siècle, le port de l'épée exprime une condition sociale plus qu'il ne constitue un caractère agressif. Si le bâton est interdit régulièrement aux $\mathrm{XVII}^{\mathrm{e}}$-XVIII ${ }^{\mathrm{e}}$ siècles, la canne est " la véritable arme du XVIII ${ }^{\mathrm{e}}$ siècle ${ }^{84}$ ". Elle est citée pour la première fois en $1685^{85}$. Parallèlement, la réglementation laisse deviner les apparitions et disparitions de certaines armes à feu : les escopettes, pétrinaux et arquebuses souvent citées dans la première moitié du $\mathrm{XVII}^{\mathrm{e}}$ siècle laissent place aux fusils par la suite. Si le pistolet est mentionné tout au long de la période, pour autant, l'arme évolue au gré des différents systèmes de mise à feu sans que la cour s'attarde sur ces précisions. Depuis le règne de Louis XIV, les armes dites "secrètes " telles que les pistolets de poche ${ }^{86}$, les baïonnettes, poignards, cannes-épées, sont plus souvent condamnées par la législation royale ${ }^{87}$ et la réglementation parlementaire qui lui a emboîté le pas. Ces armes sont interdites à tous les sujets, y compris aux nobles. Si leur port est plus souvent prohibé au XVIII ${ }^{\mathrm{e}}$ siècle, ce n'est pas tout à fait un hasard. Pour faire face aux interdictions qui se multiplient, les individus s'adaptent en essayant de trouver des parades grâce à des armes plus petites ou plus dissimulables.

Ainsi, la réglementation sur le port d'armes illustre brillamment l'utilisation du pouvoir de police générale par le parlement dans la province : la cour souveraine applique fidèlement la législation royale tout en imposant son autorité dans son ressort. Les dispositions prises par le parlement contre le port d'armes peuvent s'interpréter comme une réponse offensive au danger provoqué par " l'abus des armes ". Elles renvoient à l'image d'une société (urbaine en particulier) continuellement armée et dangereuse et laissent entendre dans ses arrêts les entrechoquements d'épées et les échanges de coups de feu. Le parlement se trouve donc sur un terrain de

84. DenYs, Catherine, Police et sécurité..., op. cit., p. 104.

85. Arch. dép. d'Ille-et-Vilaine, 1Bf 1440, 17 décembre 1685.

86. Arch. dép. d'Ille-et-Vilaine, 1Bf 229, 16 septembre 1608. Au début du XVII siècle, les petits pistolets sont " appelles vulgairement tue prests".

87. Voir les déclarations de 1660, 1666, 1679, 1700. 
Le parlement de Bretagne et la réglementation du port d'armes (1554-1789)

Tableau 2 - Les armes mentionnées dans les arrêts interdisant le port d'armes

\begin{tabular}{|c|c|c|c|c|c|c|}
\hline Armes & $<1600$ & $\begin{array}{l}1600- \\
1650\end{array}$ & $\begin{array}{l}1650- \\
1700\end{array}$ & $\begin{array}{l}1700- \\
1750\end{array}$ & $>1750$ & Total (\%) \\
\hline arme à feu & 4 & 51 & 29 & 18 & 19 & $121(34,2 \%)$ \\
\hline armes à feu & & 12 & 5 & 7 & 1 & $25(7,1 \%)$ \\
\hline arquebuse & 2 & 13 & 2 & & 1 & $18(5,1 \%)$ \\
\hline carabine & 2 & 6 & 2 & & 1 & $8(2,3 \%)$ \\
\hline escopette & & 4 & 1 & & & $5(1,4 \%)$ \\
\hline fusil & & 1 & 9 & 3 & 12 & $25(7,1 \%)$ \\
\hline mousquet & & 1 & & & & $1(0,3 \%)$ \\
\hline pistolet & 2 & 11 & 10 & 5 & 4 & $32(9,0 \%)$ \\
\hline pétrinal & & 2 & & & & $2(0,6 \%)$ \\
\hline pistolet de poche & & 1 & & 3 & 1 & $5(1,4 \%)$ \\
\hline arme blanche & 2 & 41 & 19 & 26 & 35 & $123(34,7 \%)$ \\
\hline baïonnette & & & 2 & 3 & & $5(1,4 \%)$ \\
\hline couteau & & & & 2 & 2 & $4(1,1 \%)$ \\
\hline couteau de chasse & & & & & 2 & $2(0,6 \%)$ \\
\hline coutelas & & & & & 1 & $1(0,3 \%)$ \\
\hline dague & & 5 & & & 1 & $6(1,7 \%)$ \\
\hline poignard & & 10 & 2 & 4 & 1 & $17(4,8 \%)$ \\
\hline épée & & 23 & 15 & 17 & 24 & $79(22,3 \%)$ \\
\hline espadon & & 2 & & & & $2(0,6 \%)$ \\
\hline fléau & & 1 & & & & $1(0,3 \%)$ \\
\hline hallebarde & 1 & & & & & $1(0,3 \%)$ \\
\hline pique & 1 & & & & & $1(0,3 \%)$ \\
\hline sabre & & & & & 4 & $4(1,1 \%)$ \\
\hline arme de trait & & 2 & 1 & & & $3(0,8 \%)$ \\
\hline arbalète & & 2 & 1 & & & $3(0,8 \%)$ \\
\hline armure & 2 & & & & & $2(0,6 \%)$ \\
\hline brigandine & 1 & & & & & $1(0,3 \%)$ \\
\hline maille & 1 & & & & & $1(0,3 \%)$ \\
\hline artillerie & & & & & 2 & $2(0,6 \%)$ \\
\hline canon & & & & & 2 & $2(0,6 \%)$ \\
\hline bâton / canne & & 16 & 12 & 13 & 35 & $76(21,5 \%)$ \\
\hline bâton & & 16 & 9 & 7 & 18 & $50(14,1 \%)$ \\
\hline bâton ferré & & & & & 1 & $1(0,3 \%)$ \\
\hline canne & & & 1 & 6 & 16 & $23(6,5 \%)$ \\
\hline crosse & & & 2 & & & $2(0,6 \%)$ \\
\hline non indiqué & 2 & 12 & 5 & 3 & 5 & $27(7,6 \%)$ \\
\hline armes & 2 & 12 & 5 & 3 & 5 & $27(7,6 \%)$ \\
\hline Total général & 10 & 122 & 66 & 60 & 96 & $354(100,0 \%)$ \\
\hline
\end{tabular}


lutte incessant qui ne vise qu'à une chose : maintenir l'ordre public, de jour comme de nuit, grâce à une panoplie répressive qui tend à s'adoucir. Interdit aux roturiers mis à part quelques exceptions ponctuelles et encadrées, le port d'armes est réservé aux nobles, militaires et officiers royaux avec toutes les nuances qu'il convient d'ajouter selon le type d'armes, les lieux, les époques et les moments de la journée. Contrairement à ce qu'on aurait pu penser, les arrêts du premier $\mathrm{XVII}^{\mathrm{e}}$ siècle sont ceux qui offrent le plus de détails sur la mobilisation armée des habitants pour les arrestations, sur les désarmements, les peines, etc. contrairement à ceux du XVIII ${ }^{\mathrm{e}}$ siècle où les formules stéréotypées sont plus largement utilisées. En amont, c'est toute une culture juridique commune, héritée de la fin du Moyen Âge qui transparaît dans les interdictions. À travers cette étude, on mesure à quel point le port d'armes demeure un usage difficile à endiguer, car la détention d'armes est autorisée en vertu du principe d'autodéfense. Le cœur de cette ambiguïté est d'autoriser la possession d'armes tout en interdisant d'en porter. Ainsi, les arrêts sur le port d'armes permettent de s'interroger sur le décalage existant entre la vision d'une société armée que ces derniers laissent percevoir et le véritable armement de la population qui tend à s'amenuiser au cours de l'Ancien Régime. 


\section{RÉSUMÉ}

Tout au long de l'Ancien Régime, l'interdiction du port d'armes constitue un des moyens utilisés pour endiguer les violences armées dans le royaume. À l'échelle de la Bretagne, la prérogative revient rapidement au parlement qui énonce aux $\mathrm{XVII}^{\mathrm{e}}$ et $\mathrm{XVIII}{ }^{\mathrm{e}}$ siècles, grâce à son pouvoir de police générale, une réglementation continue pour faire respecter la prohibition. Cet article se propose d'étudier les efforts croissants menés par la cour pour réprimer l'usage du port d'armes dans l'espace public. Les villes, et en premier lieu Rennes, y prennent toute leur place, témoignant du danger du milieu urbain, propice à un déploiement de la violence armée. Les dégâts causés par le braconnage ne font pas oublier pour autant les campagnes. Si le port d'armes est majoritairement interdit aux roturiers, ceux-ci bénéficient d'autorisations ponctuelles et encadrées en fonction des contextes. Le droit de porter des armes qui revient en principe aux nobles, officiers et militaires n'est pas non plus une évidence car le danger des armes à feu et des armes " secrètes " conduit la cour à prononcer des interdictions valables pour tous les sujets. Par le biais des 161 arrêts promulgués entre 1554 et 1789, se révèlent alors les enjeux de la réglementation du port d'armes pour le parlement : contrôler la population et maintenir l'ordre public. Des fers qui s'entrechoquent aux échanges de coups de feu, en passant par les rixes à coups de bâtons, il est possible à travers les arrêts sur remontrance, d'étudier les armes condamnées par le parlement et le lexique employé pour qualifier l'incrimination.

\section{ABSTRACT}

Throughout the Ancien Régime, the ban on carrying weapons was one of the ways used to curb armed violence in the kingdom. In Brittany, the parlement was made responsible for controlling violence. During the seventeenth and eighteenth centuries, it enacted a series of regulations to uphold the ban. This article will analyse the increasing efforts of the court to prohibit the use of weapons in public places. Cities, and in particular Rennes, contributed to this effort, illustrating the danger of an urban environment likely to favour the spread of armed violence. The damage caused by poaching also emphasised the importance of the countryside. Even if commoners were normally forbidden from carrying weapons, they were occasionally allowed to in special circumstances and under supervision. The right to bear arms which was normally attributed to nobles, officers and military men was not automatic, not least because the danger of firearms and "secret" weapons that led the parlement to issue a blanket ban. Thanks to the 161 judgments promulgated by the parlement between 1554 and 1789, the stakes of regulating weapons are made abundantly clear. The goal was to control the population and maintain public order. From swords clinking to gunshots and scuffles with sticks, thanks to the parlement's judgments it is possible to examine the types of weapons condemned by the court and the vocabulary used to describe the incriminations. 